\title{
Driver Reaction Time to Tactile and Auditory Rear-End Collision Warnings While Talking on a Cell Phone
}

\author{
Rayka Mohebbi and Rob Gray, Arizona State University, Mesa, Arizona, and \\ Hong Z. Tan, Purdue University, West Lafayette, Indiana
}

\begin{abstract}
Objective: This study examined the effectiveness of rear-end collision warnings presented in different sensory modalities while drivers were engaged in cell phone conversations in a driving simulator. Background: Tactile and auditory collision warnings have been shown to improve braking response time (RT) in rear-end collision situations. However, it is not clear how effective these warnings are when the driver is engaged in attentionally demanding secondary tasks, such as talking on a cell phone. Method: Sixteen participants in a driving simulator experienced three collision warning conditions (none, tactile, and auditory) in three conversation conditions (none, simple hands free, complex hands free). Driver RT was captured from warning onset to brake initiation (WON2B). Results: WON2B times for auditory warnings were significantly larger for simple conversations compared with no conversation $(+148 \mathrm{~ms})$, whereas there was no significant difference between these conditions for tactile warnings $(+53 \mathrm{~ms})$. For complex conversations, WON2B times for both tactile $(+146 \mathrm{~ms})$ and auditory warnings (+221 ms) were significantly larger than during no conversation. During complex conversations, tactile warnings produced significantly shorter WON2B times than no warning $(-141 \mathrm{~ms})$. Conclusion: Tactile warnings are more effective than auditory warnings during both simple and complex conversations. Application: These results indicate that tactile rear-end collision warnings have the potential to offset some of the driving impairments caused by cell phone conversations.
\end{abstract}

\section{INTRODUCTION}

Rear-end collisions are the most common type of accident involving another vehicle, accounting for more than $30 \%$ of such accidents (National Highway Traffic Safety Administration, 2006). Driver inattention has been reported as the most common cause: It has been estimated that more than $60 \%$ of rear-end collisions are caused by inattentive drivers (Knipling et al., 1993; Lee, McGehee, Brown, \& Reyes, 2002). Following too closely is the other major contributor, and together, driver inattention and following too closely are present in almost $90 \%$ of all rearend crashes (Knipling et al., 1993).

Recently, efforts have been put into developing rear-end collision warnings that capture the driver's attention when a collision is imminent. One important question that has arisen out of this line of research is, What is the best sensory modality in which to present a warning to the driver? Although previous studies showed a significant reduction of rear-end collisions with audiovisual warnings (e.g., Bhatia, 2003; Lee et al., 2002) and audio warnings (Abe \& Richardson, 2005), it has been proposed that these modalities may not be optimal for driver warnings because they are already very much engaged in the driving task (Belz, Robinson, \& Casali , 1999; Lee, Hoffman, \& Hayes, 2004). For example, even drivers who are actively engaged in the driving task are likely to miss visual warnings if their attention is not forward. Alternatively, a visual warning display may place demands on visual attention that compete with those required for the detection of an impending collision (Hirst \& Graham, 1997). Similarly, auditory stimuli in 
the driving environment (e.g., music, cell phone conversations, conversations with passengers, voice-activated navigation displays) may sufficiently load the auditory system and thereby limit the effectiveness of auditory collision warnings and/or mask the warning signal.

As an alternative, it has been suggested that tactile warnings may be more effective in preventing rear-end collisions because this sensory modality is relatively unengaged during driving. Although it has been shown that tactile warnings delivered via the gas pedal (Godthelp \& Schuman, 1993) or small vibrating tactors embedded in the driver's safety restraint or seat (Ho, Reed, \& Spence, 2006; Ho, Tan \& Spence, 2005; Van Erp, 2005) can also be used effectively to alert the driver to an impending rearend collision, none of these previous studies directly compared all of the different warning modalities.

To address the question of what is the most effective sensory modality to present collision warnings, Scott and Gray (2008) directly compared visual, auditory, and tactile collision warnings with a no-warning condition in a driving simulator. The warnings in all modalities were designed to meet the guidelines for optimal warnings proposed by McGehee, LeBlanc, Kiefer, and Salinger (2002). Scott and Gray (2008) found that both auditory and tactile warnings produced significantly faster brake reaction times (RT) in response to a collision event than either no warning or the visual warning. There was no significant difference between the brake RTs to auditory and tactile warnings. However, in this study, the only auditory stimuli other than the warning signal was music played through the car radio. In real driving, there are frequently additional sources of auditory information - in particular, cell phone conversations.

Would the addition of a cell phone conversation reduce the effectiveness of auditory collision warnings? Results from the majority of simulator and closed-track studies on cell phone use during driving (reviewed in Horrey \& Wickens, 2006) suggest that this may indeed be the case, given that it has been demonstrated that cell phone conversations can significantly increase driver workload. This result would also be predicted by the multiple resource theory of attention (Wickens, 1980). Crash data from recent naturalistic driving studies indicate that the risk ratio during cell phone conversations is not significantly different than when driving alone (Dingus et al., 2006), which implies that talking on a cell phone either is typically a lowload or is a high load-task that leads to adaptations in driver behavior (e.g., slowing down).

The primary goal of the present study was to examine the relative effectiveness of auditory and tactile rear-end collision warnings during simulated cell phone conversations to determine if such warnings can offset some of the observed impairments caused by cell phone use (e.g., Strayer \& Drews, 2007). From multiple resource theory (Wickens, 1980), we predicted that the additional auditory load of a cell phone conversation would lead to significantly shorter RTs for tactile rear-end collision warnings compared with auditory warnings. Because the complexity of the cell phone conversation has been shown to be an important mediating factor in impairments in driving performance (Horrey \& Wickens, 2006), we also examined the effectiveness of these collision warnings for both simple and complex conversations.

\section{METHOD}

\section{Driving Simulator and Warnings}

The DS-600c Advanced Research Simulator by DriveSafety was used. This simulator comprised a $300^{\circ}$ wraparound display, a full-width automobile cab (a Ford Focus), and a motion platform. Tactile and proprioceptive feedback cues were provided via dynamic torque feedback from the steering wheel and vibration transducers mounted under the driver's seat. The motion platform provided coordinated inertial cues for the onset of longitudinal acceleration and deceleration. The DriveSafety software captured various driving performance elements at $60 \mathrm{~Hz}$.

The auditory warnings were identical to those used in our previous study, which compared the effects of warning modality (Scott \& Gray, 2008). The auditory warning was a 75-dB, $2,000-\mathrm{Hz}$ auditory tone issued from an array of three 6.5-cm-diameter speakers (with lateral separation of $10 \mathrm{~cm}$ center to center) located on the dashboard. The speakers were located on the 
dashboard $9^{\circ}$ to $12^{\circ}$ below the driver's line of sight. The center speaker was located directly in front of the driver.

This warning was designed to follow the guidelines of McGehee et al. (2002), who recommended a "distinctive, non-speech auditory warning ... that emanates from the general direction of the threat" (p. 6). The 75-dB intensity of the auditory warning was also chosen based on the McGhee et al. guidelines and was considerably greater than the combined intensity of the noise from the simulated engine, road, traffic, and radio (approximately $60 \mathrm{~dB}$ ). We chose $2,000 \mathrm{~Hz}$ for the warning because it is within the range of frequencies that produce the lowest detection thresholds (Goldstein, 2006). We were not concerned with the driver's ability to localize the auditory signal because we did not use directional warnings in the present study.

To ensure that all participants could hear the auditory warning, we performed a screening test prior to data collection. In this test, warnings were presented at random intervals during a practice drive (with no lead vehicle), and participants were instructed to indicate verbally when they heard a warning sound. All participants had a detection rate of $100 \%$.

The tactile warning was delivered via three tactors $(2.54 \times 1.85 \times 1.07 \mathrm{~cm}$; VBW32, Audiological Engineering Corp., Somerville, MA) driven by a $290-\mathrm{Hz}$ sinusoidal signal at an intensity sufficient to deliver clearly perceptible vibrotactile stimuli. The tactors were mounted in a soft housing to mask the audio output from the activated tactors. The tactor housing was fastened on a waist belt over the driver's clothing and positioned on the center front of the driver's abdomen. This tactor positioning is identical to that used in previous experiments comparing collision warnings in different modalities (e.g., Ho, Reed, \& Spence, 2007). The amplitude of the tactile simulation was approximately $15.5 \mathrm{~dB}$ above human detection threshold for the lower abdomen (Bolanowski, Gescheider, Verrillo, \& Checkosky, 1988).

When triggered (detailed later), both tactile and auditory warnings activated for $200 \mathrm{~ms}$ with an 800-ms pause (i.e., once per second for $200 \mathrm{~ms}$ ) and continued until the car moved time to collision (TTC) warning zone of less than 5.0 $\mathrm{s}$ or until a collision occurred.

Hands-free cell phone conversations were simulated via the use of two sets of headphones with attached microphones (Logitech Model 980369). The two sets of headphones were connected via a computer network with Skype software. The experimenter conversed with the participant from a separate, closed room and was not visible to the participant.

\section{Design and Procedure}

Sixteen drivers between the ages 19 and 49 $(M=26.2, S D=9.1)$ participated in the study. There were 9 females and 7 males. All participants were licensed drivers with between 3 and 26 $(M=8.8, S D=5.2)$ years of driving experience. All drivers completed an informed consent form and were compensated for their participation. The drivers were naive regarding the aims of the experiment.

Participants followed a red lead car on a rural, two-lane road and were instructed to drive in their own lane and not to pass the lead car. They were given two different 5-min practice drives without any warning or conversations that would enable them to become familiar with the driving simulator. Drivers were permitted to repeat the practice if requested. The first practice drive merely familiarized them with driving in the simulator, and in the second drive, they familiarized themselves with the driving scenarios. For both the practice and experimental sessions, drivers were instructed to maintain a 2.0-s TH with the lead car (Ho et al., 2006). If the driver followed too far behind the lead car, the words Speed Up! would appear in red text on the driver's display. There was no analogous Slow Down! warning, so drivers were free to maintain any $\mathrm{TH}$ of less than $2.0 \mathrm{~s}$.

In the experimental sessions, the collision warning activated when the time-to-collision (TTC; D. N. Lee, 1976) between the driver's vehicle and the lead car fell below a critical threshold of $5.0 \mathrm{~s}$. This $5.0-\mathrm{s}$ value was chosen because it proved to be most effective for collision warnings in our previous study (Scott \& Gray, 2008). The lead car was programmed to change speeds unpredictably (to the driver) at variable intervals. The lead car traveled between 
$55 \mathrm{mph}$ and $65 \mathrm{mph}$ (with an average speed of $60 \mathrm{mph}$ ); its speed variation was determined by a sum of three sinusoids. The lead car was programmed to make eight unpredictable (to the driver) full stops at $-6 \mathrm{~m} / \mathrm{s}^{2}$. The unpredictable behavior of the lead car made it very difficult for the driver to predict when the lead car would speed up, slow down, or stop, creating multiple possible rear-end collision situations.

The brake lights of the lead car were disabled so the present results would be directly comparable with those of previous studies in this area (Ho et al., 2006; Scott \& Gray, 2008). A control experiment in our previous study indicated that the presence or absence of brake lights does not alter the relative effectiveness of the different warnings (Scott \& Gray, 2008; see also Ho et al., 2007).

Intermittent opposing roadway traffic was included to more closely simulate real-world rural driving conditions. For compatibility purposes, the present experiment included the same driving scenarios and tracks used by Scott and Gray (2008). If the participant contacted the lead vehicle (i.e., crashed), an audio file of a crash sound was presented for a duration of $500 \mathrm{~ms}$, and the lead vehicle disappeared from the screen. The participant was then instructed to catch up with the next lead vehicle on the road.

Each participant completed nine driving tracks corresponding to nine experimental conditions: (a) no conversation-no warning, (b) no conversation-tactile warning, (c) no conversationaudio warning, (d) simple conversation-no warning, (e) simple conversation-tactile warning, (f) simple conversation-audio warning, (g) complex conversation-no warning, (h) complex conversation-tactile warning, and (i) complex conversation-audio warning. Each track had eight unpredictable full stops of the lead car and required roughly 5 to 6 min to complete. The order of these nine conditions was counterbalanced across participants. Participants received a 5-min rest between conditions to minimize simulator sickness and fatigue and to allow the experimenter to set up the next driving scenario.

In the simple conversation conditions, the conversation between the participant and experimenter
TABLE 1: Questions in the Simple Conversation Condition

\begin{tabular}{ll}
\hline No. & \multicolumn{1}{c}{ Question } \\
\hline 1 & What is your major? What do you do? \\
2 & Where were you born? If not Arizona, \\
3 & how did you end up in Arizona? \\
& Are you married and do you have \\
4 & Did you have a nice summer? Did you \\
5 & go anywhere fun? \\
& Do you like to go to the movie \\
& theater? What is the latest movie \\
7 & Which have seen? \\
8 & What sport do you like to play? \\
& What car do you drive? Do you \\
\end{tabular}

consisted of a series of scripted demographic and personal questions shown in Table 1. In the complex conversation conditions, the conversation between the participant and experimenter consisted of a series of mental math and categorization questions, similar to the method used by Paten, Kirscher, Ostlund, and Nilsson (2004). For each question, participants were required to perform single-digit addition, categorize the result as even or odd, and then remember the last number read by the experimenter for the next addition task. An example follows:

Experimenter: Three, six.

Driver: Three plus six equals nine; odd.

Experimenter: Four.

Driver: Six plus four equals ten; even.

\section{Data Analysis}

The main dependent variable was the driver brake RT for each lead car stopping event (WON2B). WON2B was defined as the elapsed time between the onset of the collision warning (i.e., TTC $=5.0 \mathrm{~s}$ ) and brake onset. Only stopping events for which the driver received a warning were included in the analysis (e.g., we discarded events for which the driver was too far away from the lead car at the time of stopping to receive a warning). We also discarded 


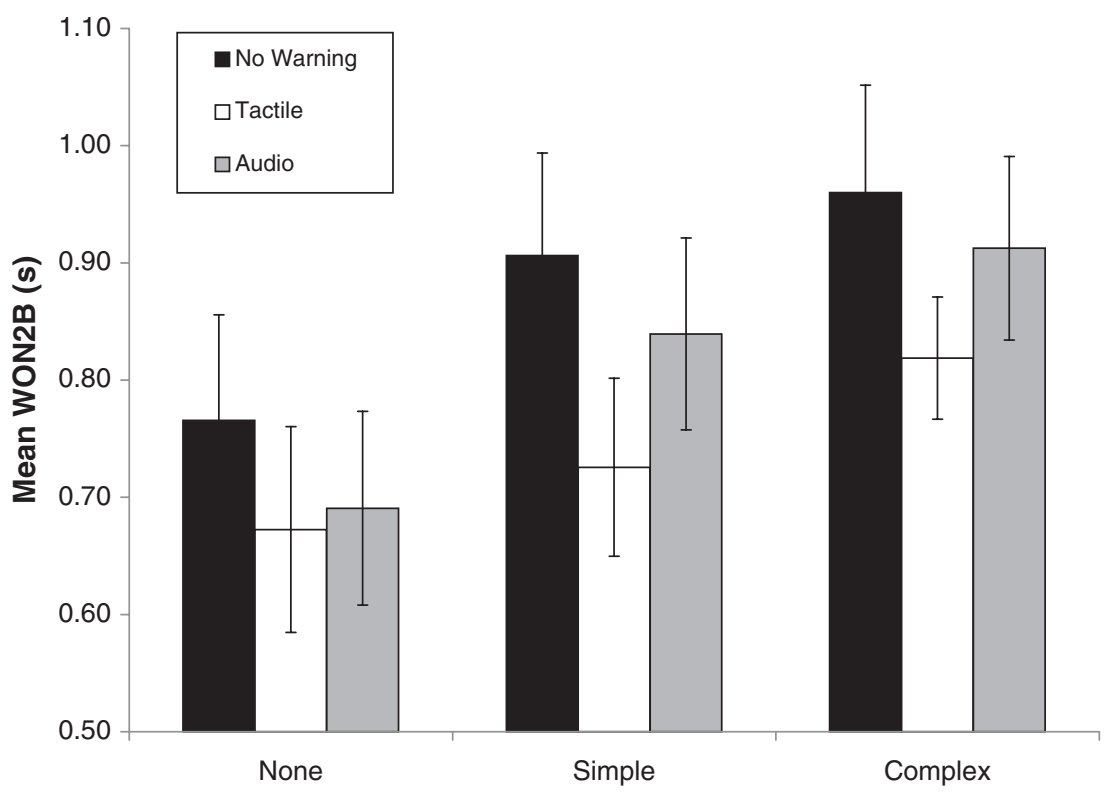

Conversation Type

Figure 1. Mean times from warning onset to brake initiation. Error bars are standard errors.

any events for which the WON2B time was less than $350 \mathrm{~ms}$ (see Scott \& Gray, 2008) or more than $3 \mathrm{~s}$. This range was chosen to remove trials for which the driver was already initiating a braking response when the warning was initiated and trials for which the driver made no response to the warning. The number of collision events discarded per driver ranged between 5 and 9 of the total possible 72 events. There was no significant effect of conversation type or warning type on the number of valid events. Note that in the no-warning conditions, WON2B refers to the time elapsed between when the TTC reached the threshold of $5 \mathrm{~s}$ and the onset of braking.

We first analyzed mean WON2B times (averaged across all of the stopping events for each driver) using a $3 \times 3$ repeated-measures ANOVA with conversation type (none, simple, and complex) and warning type (none, tactile, and audio). We further analyzed these data using a set of eight single $d f$ planned contrasts corresponding to the available $d f$ in our $3 \times 3$ design:

1. no conversation-no warning versus simple conversation-no warning

2. no conversation-tactile versus simple conversation-tactile
3. no conversation-audio versus simple conversationaudio

4. no conversation-no warning versus complex conversation-no warning

5. no conversation-tactile versus complex conversation-tactile

6. no conversation-audio versus complex conversation-audio

7. complex conversation-no warning versus complex conversation-tactile

8. complex conversation-no warning versus complex conversation-audio

We also analyzed the percentage of collisions (i.e., number of collisions divided by total valid braking events) using the same ANOVA and set of planned contrasts.

\section{RESULTS}

Figure 1 plots the mean WON2B times (i.e., the mean of the mean WON2B for all drivers) for the nine experimental conditions. The overall ANOVA revealed significant main effects of conversation type, $F(2,30)=66.9, p<.001$, and warning type, $F(2,30)=46.1, p<.001$, and a significant Conversation Type $\times$ Warning Type interaction, $F(4,60)=2.85, p<.05$. 
TABLE 2: Mean Percentage of Collisions

\begin{tabular}{lcc}
\hline Condition & Mean \% & Standard Deviation \\
\hline No conversation-no warning & 7.8 & 1.9 \\
No conversation-tactile & 4.7 & 2.3 \\
No conversation-audio & 5.6 & 1.7 \\
Simple conversation-no warning & 8.0 & 2.1 \\
Simple conversation-tactile & 5.1 & 2.2 \\
Simple conversation-audio & 7.7 & 1.5 \\
Complex conversation-no warning & 8.8 & 2.6 \\
Complex conversation-tactile & 6.3 & 1.8 \\
Complex conversation-audio & 8.3 & 2.0 \\
\hline
\end{tabular}

We further analyzed these effects using the eight planned contrasts described earlier. Planned Contrasts 1 through 3 revealed that the addition of the simple conversation significantly increased mean WON2B times (compared with no conversation) for both no warnings, $t(30)=-4.5, p<.001$, and audio warnings, $t(30)=-7.8, p<.001$, whereas there was no significant change for tactile warnings, $t(30)=-1.8, p>.05$. Planned Contrasts 4 through 6 revealed that the addition of the complex conversation significantly increased mean WON2B times (compared with no conversation) for all three warning types: no warning, $t(30)=-6.1, p<.001$; tactile, $t(30)=-5.7, p<$ .001 ; and audio, $t(30)=-7.8, p<.001$. Planned Contrast 7 revealed that mean WON2B times in the complex condition were significantly lower for tactile warnings than for no warning, $t(30)=5.4, p<.001$. Finally, Planned Contrast 8 revealed that mean WON2B times in the complex condition were not significantly different for audio and no warnings, $t(30)=1.6, p>.05$.

The mean percentage of collisions for the nine experimental conditions are shown in Table 2. Although adding simulated cell phone conversations did increase the percentage of collisions, none of the main effects or interactions in the ANOVA, nor any of the planned comparisons, performed on collision percentage data was significant.

There were no significant correlations between WON2B (or percentage of collisions) and the following self-reported driver demographic variables: age, gender, video gaming frequency, years of licensed driving experience, and frequency of cell phone use while driving.

\section{DISCUSSION}

The primary aims of the present study were to determine (a) if the consistent (but not significant) advantage for tactile rear-end collision warnings compared with auditory warnings reported by Scott and Gray (2008) would increase with the addition of simulated cell phone conversations and (b) whether auditory and tactile rear-end collision warnings can offset some of the impairments in brake RTs caused by cell phone conversations of differing levels of complexity.

As shown in Figure 1, the present findings provide support for our prediction that an increase in the load on the auditory system produced by simple, casual cell phone conversations would cause tactile rear-end collision warnings to be significantly more effective than auditory rearend collision warnings, as evidenced by shorter brake RTs. Whereas the addition of a simple conversation in the auditory warning condition leads to significantly slowed brake RTs relative to no conversation (by $148 \mathrm{~ms}$ on average, corresponding to a distance of $3.6 \mathrm{~m}$ for a speed of $55 \mathrm{mph}$ ), cell phone conversations did not produce a significant slowing of brake RTs when tactile rear-end collision warnings were used. We would argue that these results are generally consistent with the multiple resource theory of attention (Wickens, 1980), which hypothesizes that people have separate pools of attentional resources for the different sensory modalities. 
These findings suggest that tactile rear-end collision warnings could reduce the impairments in driver RT caused by simple conversations on cell phones while driving (e.g., Strayer \& Drews, 2007), whereas auditory warnings will be relatively ineffective. Consistent with previous simulator studies examining hands-free cell phone use (reviewed in Horrey \& Wickens, 2006), brake RT in the no-warning condition was significantly elevated for simple conversations compared with no conversation (by $140 \mathrm{~ms}$, on average).

A similar pattern of results was found for the complex conversations used in the present study. In this condition, the addition of cell phone conversation again significantly increased brake RT for auditory warnings relative to no conversation (by $221 \mathrm{~ms}$ on average, corresponding to a distance of $5.4 \mathrm{~m}$ for a speed of $55 \mathrm{mph}$ ). As shown in Figure 1, the addition of the complex conversation did significantly reduce the effectiveness of tactile warnings compared with no conversation: Brake RT increased by $146 \mathrm{~ms}$ on average, corresponding to a distance of $3.6 \mathrm{~m}$ for a speed of $55 \mathrm{mph}$. However, in the complex conversation condition, brake RTs for the tactile warning were still significantly shorter (by $141 \mathrm{~ms}$ on average) than brake RT for no warnings. This was not the case for audio warnings, as there was no significant difference between audio warnings and no warnings in the complex conversation condition (mean difference $=47 \mathrm{~ms}$ ).

Together, the results of the present study imply that tactile collision warnings will be more effective than auditory collision warnings in the presence of cell phone conversations of any level of complexity. However, two important issues must be considered before this conclusion can be made. The first is the relative salience of the warnings. Although the auditory and tactile warnings used in our study were specifically designed to have similar signal intensities (relative to detection thresholds for each modality), it is possible that they were not of equal salience. For example, the tactile warnings may have been more "attention grabbing" than the auditory warnings. One concern that was raised with our previous study (Scott \& Gray, 2008) was that there was a difference in masking - the auditory warning was presented over a background of music and engine noise, whereas there were no other vibratory signals besides the tactile warning. In a pilot study, we found no significant difference between the relative effectiveness of the auditory and tactile warnings for fixed-base and motion-base (which did provide vehicle vibration) driving simulators, suggesting that differential masking cannot fully explain the present findings.

The salience of the two warnings may also be influenced by the location of the signal source relative to the collision event. Studies on crossmodal attentional cuing (e.g., Spence \& Driver, 1997) have shown that RTs to a visual target are faster when the cue (either tactile or auditory) is in close spatial proximity to the location of the target stimulus. However, in the present study, this effect would presumably be greater for the auditory warnings because the speakers (mounted on the dashboard) were much closer to the location of the lead vehicle than were the tactors (mounted on the seat belt). The final point to consider is the difference in cell phone impairments reported for driving simulator and naturalistic driving studies. Because simulator studies such as ours tend to find substantially larger impairments, it would be interesting if future researchers could determine if there is still a significant advantage of tactile warnings compared with auditory and no warnings in a naturalistic setting.

The findings of the present experiment were limited by certain constraints imposed through the simulation paradigm. First, the drivers in the present experiment were fully expecting the lead car to stop suddenly, so driver responses recorded in this simulation were likely faster than can be expected in a real driving situation. However, it is reasonable to expect that the relative increase in the brake RT caused by cell phone conversions in each modality would be the same in real driving; that is, the brake RTs to tactile and audio warnings should increase to a similar extent in real-world driving situations involving cell phone use by drivers. Nonetheless, this needs to be tested empirically. Furthermore, the frequency of warnings in the present study was much higher than would occur in real driving, and it is possible that this high warning frequency led to unnatural driving 
behavior (see Lee et al., 2002). Future research is needed in which warnings occur at a much lower frequency.

Another limitation of the present study was the unnatural complex conversation used. Like many previous researchers (see Horrey \& Wickens, 2006), we employed an arithmetic task for a complex "conversation" because it could be controlled more easily in an experiment. The effects of more naturalistic complex conversations (e.g., holding a business meeting or debating an emotionally charged issue) on collision warnings in different modalities should also be investigated in future experiments. Similarly, our "simple" and "complex" conversations differed on dimensions other than cognitive load, including processing code (spatial vs. verbal) and memory load. Future research that directly manipulates these different secondary task dimensions is needed to fully understand which aspects of conversation interfere with driving performance and the ability to detect collision warnings.

\section{CONCLUSION}

Driver inattention is the most common cause of rear-end collisions. Previous research suggests that audiovisual and tactile rear-end collision warnings can be effective in directing a driver's attention to an impending collision, thus reducing the braking RT and decreasing the chance of a rear-end collision. However, an important question that has not been addressed is whether such warnings can be effective when the driver is engaged in a cell phone conversation. The present study examined the relative effectiveness of auditory and tactile rear-end collision warnings during simulated simple and complex cell phone conversations. For both simple, casual conversations that occur most frequently during everyday driving and complex, cognitively demanding conversations (e.g., negotiating a business deal), the present findings suggest that tactile warnings lead to faster brake RTs in response to a lead car's braking event than either auditory warnings or no warnings.

\section{ACKNOWLEDGMENTS}

The work presented here represents part of the master's degree requirements completed by the first author. It was supported by the National Science Foundation, Information and Intelligent Systems Program (Award No. 0533908 to the second and third authors). We thank Roger Schvaneveldt for advice on statistics.

\section{REFERENCES}

Abe, G., \& Richardson, J. (2005). The influence of alarm timing on braking response and driver trust in low speed driving. Safety Science, 43(9), 639-654.

Alm, H., \& Nilsson, L. (1995). The effects of a mobile telephone task on driver behavior in a car following situation. Accident Analysis \& Prevention, 27, 707-715.

Belz, S. M., Robinson, G. S., \& Casali, J. G. (1999). A new class of auditory warning signals for complex systems: Auditory icons. Human Factors, 41, 608-618.

Bhatia, P. (2003). Vehicle technologies to improve performance and safety (UCTC Publication No. 622). Berkeley: University of California.

Bolanowski, S. J., Gescheider, G. A., Verrillo, R. T., \& Checkosky, C. M. (1988). Four channels mediate the mechanical aspects of touch. Journal of the Acoustical Society of America, 84, 1680-1694.

Dingus, T. A., Klauser, S. G., Neale, V. L., Petersen, A., Lee, S. E., Sudweeks, J., Perez, M. A., Hankey, J., Ramsey, D., Gupta, S., Bucher, C., Doerzaph, Z. R., Jermeland, J., \& Knipling, R. R. (2006). The 100-Car Naturalistic Driving Study Phase II: Results of the 100-car field experiment (Report No. DOT HS 810593). Washington, DC: National Highway Traffic Safety Administration.

Godthelp, J., \& Schuman, J. (1993). Intelligent accelerator: An element of driver support. In A. M. Parkes \& S. Frazen (Eds.), Driving future vehicles (pp. 265-275). London: Taylor \& Francis.

Goldstein, E. B. (2006). Sensation and perception. Pacific Grove, CA: Wadsworth.

Hirst, S., \& Graham, R. (1997). The format and presentation of collision warnings. In Y. I. Noy (Ed.), Ergonomics and safety of intelligent driver interfaces (pp. 203-219). Mahwah, NJ: Erlbaum.

Ho, C., Reed, N., \& Spence, C. (2006). Assessing the effectiveness of "intuitive" vibrotactile warning signals in preventing frontto-rear-end collisions in a driving simulator. Accident Analysis \& Prevention, 38, 989-997.

Ho, C., Reed, N., \& Spence, C. (2007). Multisensory in-car warning signals for collision avoidance. Human Factors, 49, 1107-1114.

Ho, C., Tan, H. Z., \& Spence, C. (2005). Using spatial vibrotactile cues to direct visual attention in driving scenes. Transportation Research Part F: Traffic Psychology and Behaviour, 8(6), 397-412.

Horrey, W. J., \& Wickens, C. D. (2006). Examining the impact of cell phone conversations on driving using meta-analytic techniques. Human Factors, 48, 196-205.

Knipling, R. R., Mironer, M., Hendriks, D. L., Tijerina, L., Everson, J., Allen, J. C., \& Wilson, C. (1993). Assessment of IVHS countermeasures for collision avoidance: Rearend crashes (DOT HS 807 995). Washington. DC: National Highway Traffic Safety Administration.

Lee, D. N. (1976). A theory of visual control of braking based on information about time-to-collision. Perception, 5, 437-459.

Lee, J. D., Hoffman, J. D., \& Hayes, E. (2004). Collision warning design to mitigate driver distraction. In Proceedings of the SIGCHI Conference on Human Factors in Computing Systems, $6,65-72$. 
Lee, J. D., McGehee, D. V., Brown, T. L., \& Reyes, M. L. (2002). Collision warning timing, driver distraction, and driver response to imminent rear-end collisions in a high-fidelity driving simulator. Human Factors, 44, 314-334.

McGehee, D. V., LeBlanc, D. J., Kiefer, R. J., \& Salinger, J. (2002). Human factors in forward collision warning systems: Operating characteristics and user interface requirements (No. J2400). Warrendale, PA: Society of Automotive Engineers.

National Highway Traffic Safety Administration. (2006). Traffic safety facts 2006: A compilation of motor vehicle crash data from the fatality analysis reporting system and the general estimates system (NHTSA Publication No. DOT- HS-810-818) Washington, DC: U.S. Department of Transportation.

Patten, C. J. D., Kircher, A., Ostlund, J., \& Nilsson, L. (2004). Using mobile telephones: Cognitive workload and attention resource allocation. Accident Analysis \& Prevention, 36, 341-350.

Scott, J. J., \& Gray, R. (2008). Comparison of visual, auditory and tactile warnings for rear-end collision prevention in simulated driving. Human Factors, 50, 264-275.

Spence, C., \& Driver, J. (1997). Cross-modal links in attention between audition, vision, and touch: Implications for interface design International Journal of Cognitive Ergonomics, 1, 351-373.

Strayer, D. L., \& F. A. Drews (2007). Cell-phone-induced driver distraction. Current Directions in Psychological Science, 16, 128-131.

Van Erp, J. B. F. (2005). Presenting directions with a vibrotactile torso display. Ergonomics, 48, 302-313.
Wickens, C. D. (1980). The structure of attentional resources. In R. S. Nickerson (Ed.), Attention and performance (pp. 239254). Mahwah, NJ: Lawrence Erlbaum.

Rayka Mohebbi is a research scientist at the Air Force Research Laboratory's Warfighter Training Research Division in Mesa, Arizona. She obtained her MS in applied psychology from Arizona State University in 2008.

Rob Gray is an associate professor in the Department of Applied Psychology at Arizona State University in Mesa, Arizona. He obtained his $\mathrm{PhD}$ in psychology from York University in 1998.

Hong Z. Tan is an associate professor in the School of Electrical and Computer Engineering at Purdue University in West Lafayette, Indiana. She obtained her $\mathrm{PhD}$ in electrical engineering and computer science from Massachusetts Institute of Technology in 1996.

Date received: March 22, 2008

Date accepted: January 31, 2009 